\title{
Nodding syndrome in Tanzania may not be associated with circulating anti-NMDA- and anti-VGKC receptor antibodies or decreased pyridoxal phosphate serum levels-a pilot study
}

\author{
Anelia Dietmann ${ }^{1}$, Bernd Wallner ${ }^{1}$, Rebekka König ${ }^{1}$, Katrin Friedrich ${ }^{1}$, Bettina Pfausler ${ }^{1}$, \\ Florian Deisenhammer ${ }^{1}$, Andrea Griesmacher ${ }^{2}$, Christoph Seger ${ }^{2}$, William Matuja ${ }^{3}$, \\ Louise JilekAall ${ }^{4}$, Andrea S. Winkler ${ }^{5}$, Erich Schmutzhard ${ }^{1}$
}

1. Department of Neurology, Medical University Innsbruck, Austria

2. Central Institute of Medical and Chemical Laboratory Diagnostics, Medical University Innsbruck, Austria

3. Division of Neurology, Muhimbili Medical Centre, Dar es Salaam, Tanzania

4. Department of Psychiatry, University of British Columbia, Vancouver, Canada

5. Department of Neurology, Technical University Munich, Germany

\begin{abstract}
Background: Nodding syndrome (NS) is a seemingly progressive epilepsy disorder of unknown underlying cause. We investigated association of pyridoxal-phosphate serum levels and occurrence of anti-neuronal antibodies against $\mathrm{N}$ methyl-D-aspartate (NMDA) receptor and voltage gated potassium channel (VGKC) complex in NS patients.

Methods: Sera of a Tanzanian cohort of epilepsy and NS patients and community controls were tested for the presence of anti-NMDA-receptor and anti-VGKC complex antibodies by indirect immunofluorescence assay. Furthermore pyridoxal-phosphate levels were measured.

Results: Auto-antibodies against NMDA receptor or VGKC (LG1 or Caspr2) complex were not detected in sera of patients suffering from NS ( $n=6)$, NS plus other seizure types $(n=16)$, primary generalized epilepsy $(n=1)$ and community controls without epilepsy $(\mathrm{n}=7)$. Median Pyridoxal-phosphate levels in patients with NS compared to patients with primary generalized seizures and community controls were not significantly different. However, these median pyridoxalphosphate levels are significantly lower compared to the range considered normal in Europeans.

Conclusions: In this pilot study NS was not associated with serum anti-NMDA receptor or anti-VGKC complex antibodies and no association to pyridoxal-phosphate serum levels was found.
\end{abstract}

Key words: nodding syndrome, epilepsy, anti-neuronal antibodies, pyridoxal-phosphate

African Health Sciences 2014; 14(2):434-438

DOI: http://dx.doi.org/10.4314/ahs.v14i2.20

\section{Introduction}

Nodding syndrome (NS) is a distinctive epilepsy disorder leading to a progressive encephalopthy with severe cognitive decline and neurological impairment in some children with appearance in geographically localized areas in South Sudan [1-3], southern Tanzania [4 7], and northern Uganda [8-11]. Nodding attacks are clinically characterized by loss of neck muscle tone and consequently

vertical head nodding, often accompanied by other

\section{Corresponding Author: \\ Anelia Dietmann, Department of Neurology \\ Innsbruck Medical University \\ Anichstrasse 35 \\ 6020 Innsbruck, Austria, anelia.dietmann@gmail.com}

types of epileptic seizures and impaired consciousness $[2,5,6,9,1214]$. NS is variably associated with growth retardation, malnutrition, delayed sexual development and cognitive impairment $[2,3,5,9,12]$. The cause of this epilepsy disorder is unknown, association with parasitic infection such as Onchocerca volvulus and Mansonella perstans as well as toxic exposures and nutritional deficiencies, especially pyridoxine (vitamin B6) have been proposed [2,5,9,1518]. Magnetic resonance imaging (MRI) showed brain atrophy in some patients in a study by Sejvar et al.[9], whereas gliotic changes and hippocampal pathologies were described by Winkler et al. [5,17]. The same study showed familial clustering and interictal epileptic activity such as intermittent generalized slowing and sharp wave activity suggesting an absence type epileptic nature of disease $[5,6]$, whereas EEG changes during the actual head nodding attack were classified as atonic seizures [9]. Clinical signs and symptoms of NS include impaired consciousness, tremors, drooling and inconti- 
nence of urine as well as psychiatric disturbances [2,5,9]. This clinical picture may also be seen in autoimmune encephalopathies [19] and it was therefore tempting to speculate thatNS is associated with antibodies to cellsurface proteins expressed in neurons [20]. Therefore,we investigated sera of a Tanzanian epilepsy cohort including NS patients and community controls originally collected for studies described in [21] - for the presence of anti-NMDA (N-methylDaspartate) receptor and antiVGKC (voltage gated potassium channel) complex antibodies as a pilot study. Furthermore we measured pyridoxalphosphate serum levels in a set of patients and community controls of the same Tanzanian cohort.

\section{Methods:}

The original study was conducted in The Mahenge Epilepsy Clinic, Mahenge, Tanzania. A detailed description of the study population has been described elsewhere [21]. The study had been approved by the Ethics Committee of the Muhimbili University College of Health Sciences,Dar es Salaam. Informed consent was obtained from all participants.

In brief, clinical characteristics, skin snips, blood samples were collected in patients regularly attending The Mahenge Epilepsy Clinic. Four different patient groups were defined: people with epilepsy with and without onchocerciasis and people not suffering from epilepsy with and without detection of onchocerciasis. People with epilepsy were further classified in subgroups of either primary generalized seizures, focal seizures, NS only or NS and occurrence of other epileptic seizure types [21]. According to current nomenclature study groups of patients with NS or NS and other seizures types correspond to NS and NS plus [5,14]. As control groups patients with primary generalized epilepsy (PGE) without NS and community controls (CC) without history of epileptic disorders or NS were enrolled according to the study protocol [21]. In all patients and community controls polymerase chain reaction (PCR) for Onchocerca volvulus has been performed on skin snips as described earlier in [21].

All samples (collected in 2005) were transferred to the Neurological Laboratory, Department of Neurology, Innsbruck Medical University, Austria. Samples were defrosted once for detection of IgG4 antibodies against Onchocerca volvulus [21], and were then stored at $20^{\circ} \mathrm{C}$ without further freezethaw cycles. For the current study, 30 randomly selected samples with sufficient amount of available serum of patients with
NS, NS plus, PGE only or CC were thawed once for detection of either pyridoxalphosphate or of antineuronal antibodies. Autoantibodies against NMDA receptors and VGKC complex, particularly LGI1 and Caspr2, were detected by a commercially available cellbased indirect immunofluorescence assay according to the manufacturer's instructions (www.euroimmun.de/ index.php?id $=29 \& \mathrm{~L}=1)$. The assay uses transfected immobilized HEK293 cells which are incubated with test sera at a dilution of 1:10. Secondary antihuman fluorescein isothiocyanate labeled $\operatorname{IgG}$ antibodies are used for detection of specific autoantibodies. To control for background activity nontransfected HEK293 cells were used and in each run a positive and a negative control serum was included. Evaluation was done in a blinded fashion at the Neurological Laboratory, Department of Neurology, Innsbruck Medical University, Austria.

Pyridoxine was measured as pyridoxal-phosphate, the active pyridoxal-phosphate metabolite.

Pyridoxalphosphate levels were measured in patients and community control serum samples according to routinely performed procedures in the central laboratory of the InnsbruckMedical University, Austria. An IVDCE certified HPLCFLD assay (Chromsystems, Munich, Germany) with external calibration and an internal standard was used. The assay was linear between 1.0 and $260.0 \mu \mathrm{g} / 1$, an inter-day variability of $<6.0 \%$ was observed.

Pyridoxalphosphate serum levels are given in median and range values and were compared within study groups by 1way ANOVA. Statistical significance was defined as a 2 sided $\mathrm{P}$ value $<0.05$.

\section{Results:}

Patients' demographic data are listed in Table 1. Autoantibodies against NMDA receptors or VGKC complex (LG1 or Caspr2) were not detected in serum of patients suffering from NS ( $n=6)$, NS plus $(n=16)$ or PGE $(n=1)$. In the serum of CC $(n=7)$ one sample was found positive for antiVGKC Caspr2 complex antibodies. Skin snips tested positive for onchocerciasis in $69 \%$ of NS, $81 \%$ of NS plus, $55 \%$ of PGE patients and $100 \%$ of CC (Table 1$)$.

\section{Table 1}

Numbers, sex and median age (range) of patients in different study groups of patients with nodding syndrome (NS), nodding syndrome and other epileptic disorders (NS plus), primary generalized epilepsy (PGE) and community controls (CC). 
Table 1 Demographics

Pyridoxine

n female Median Age (Range) Onchocerciasis (positive Skin snip)

$\begin{array}{lllll}\text { NS } & 10 & 5 & 14,5(12-18) & 7 \\ \text { PGE } & 10 & 4 & 14,5(12-18) & 5 \\ \text { CC } & 10 & 4 & 29,5(22-31) & 10\end{array}$

NMDA-Receptor and VGKC Complex Antibodies

n female Median Age (Range) Onchocerciasis (positive Skin snip)

\begin{tabular}{|c|c|c|}
\hline NS & 5 & 15 (13-65) \\
\hline NS plus & 16 & $17(12-30)$ \\
\hline GE 1 & 0 & 16 \\
\hline C & 2 & $50(26-65)$ \\
\hline
\end{tabular}

Median Pyridoxal-phosphate serum levels in patients with NS $(2,11 \mu \mathrm{g} / \mathrm{L}$, range $1,0-4,86 \mu \mathrm{g} / \mathrm{L}, \mathrm{n}=10)$ compared to PGE patients $(1,96 \mu \mathrm{g} / \mathrm{L}$, range $1,06,18 \mu \mathrm{g} / \mathrm{L}$, $\mathrm{n}=10)$ and $\mathrm{CC}(2,76 \mu \mathrm{g} / \mathrm{L}$, range $1,076,33 \mu \mathrm{g} / \mathrm{L}, \mathrm{n}=10)$ yielded no statistically significant difference (Figure 1). However, median pyridoxalphosphate levels are significantly lower compared to the range considered normal in Europeans $(6-18 \mu \mathrm{g} / \mathrm{L})$.

Figure 1

Serum pyridoxalphosphate levels of nodding syndrome (NS) patients, pimary generalized epilepsy patients (PGE) and community controls (CC). Boxes and whiskers, median and interquartile range. P not significant.

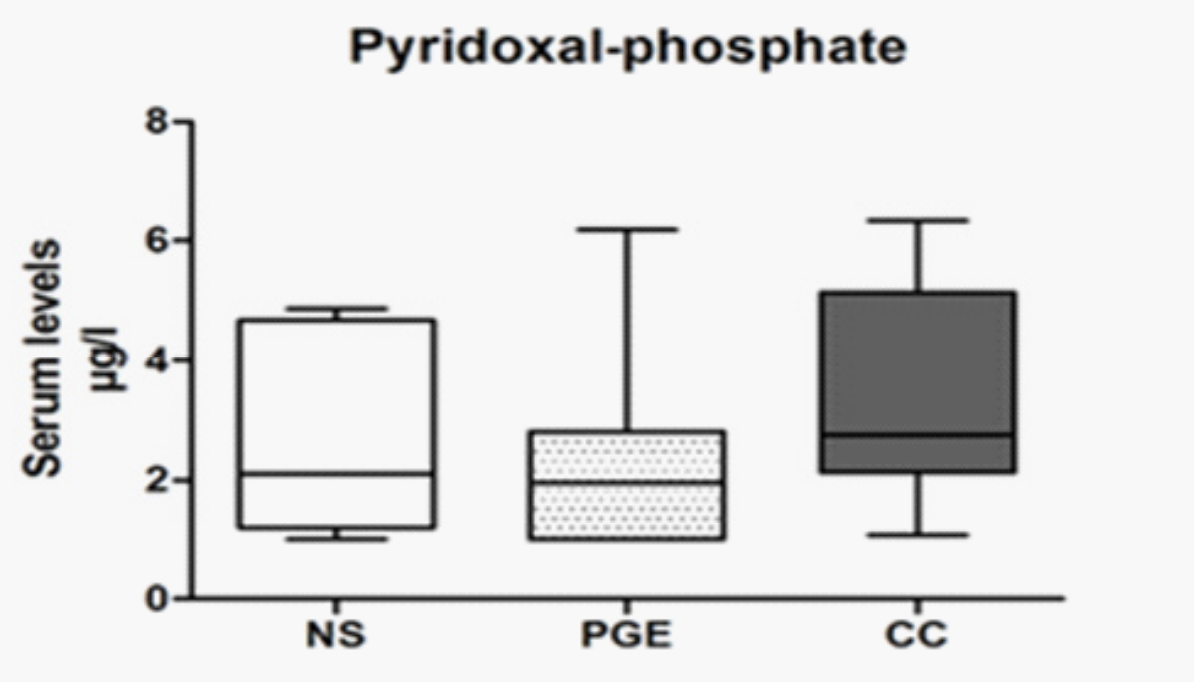

\section{Discussion:}

Serum antiNMDAreceptor or antiVGKCcomplex antibodies were not detected in NS, NS plus or PGE patients. Surprisingly we found one positive sample for antiVGKC Caspr2 complex antibodies in a community control group without epilepsy or NS in his medical history. Since this specific subject showed no signs or symptoms of any epilepsy disorder at the time of taking the serum sample and was not followed up, this finding cannot be related to any neurologic disorder of later onset.

Recently, Winkler et al. published a followup Study on NS patients from the same cohort; however community controls have not been re-examined [6]. 
MRI changes, EEG and clinical findings are associated with signs and symptoms of a progressive epileptic encephalopathy in some of the patients including impaired consciousness, tremors, drooling and incontinence of urine as well as psychiatric disturbances $[2,5,9,17]$. This clinical picture may also be seen in autoimmune encephalopathies [19] and it was therefore tempting to speculate that auto immune antibodies such as antineuronal receptor antibodies might be found circulating in the peripheral blood of NS or NS plus patients [20]. However, this is the first scientificreport to describe that anti-neuronal antibodies against NMDA-receptors or the VGKC-complex have not been found in NS or NS plus patients.

Low pyridoxalphosphate levels or other nutritional factors as well as possible exposures to toxinsare hypothesized causes for NS $[15,18]$. In the present study we measured Vitamin B6 levels of NS patients, and PGE patients and community controls. Levels did not differ significantly between these three groups. However, when compared to the range of pyridoxalphosphate serum levels considered normal in Europeans, levels were overall significantly lower. Results of low pyridoxalphosphate serum levels are consistent with findings from others described in literature [18]. This might be explained by different food habits and availability of nutrition rich in vitamin B6 or by a lower reference range for this population. In a publication by Spencer et al. environmental, nutritional and infectious factors in NS were described in detail. Spencer et al. discussed that ingestion of food contaminated by Penicillium fungi, with its metabolites acting as pyridoxine antimetabolites [22], may account for low pyridoxal-phosphate levels [16].

The current study has some important limitations. First, our sample size is small; second,other neuronal autoimmune antibodies such as antiglutamate receptor antibodies have not been investigated. Third, samples were stored for approximately 7 years before analysis. Since storage temperature was constant $\left(20^{\circ} \mathrm{C}\right)$ and freezethaw cycles were avoided, degradation of sampledproteins was likely to have been negligible.

\section{Conclusion}

In this pilot study NS and NS plus is not associated with antineuronal antibodies against NMDA receptors or VGKC complex circulating in the serum and it does not seem to be associatedwith decreased pyridoxalphosphate serum levels. Therefore, further studies are essential to elucidate the pathomechanism of this mys- terious epileptic encephalopathy affecting endemically young people in selected parts of Eastern Africa.

\section{Acknowledgments}

We thank Ingrid Gstrein from the Neurological Laboratory, Department of Neurology, Innsbruck Medical University, Austria for the performance of the cellbased indirect immunofluorescence assay.

\section{Detailed contribution of Authors to the work}

A.D., E.S. Manuscript; A.D., F.D. Cell-Based indirect immunofluorescence assays; A.G., C.S.

Measurment of Phyridoxalphoasphate levels; B.W., R.K., K.F., B.P. Patients enrollment and sample collection; A.S.W., W.M., L.J.A., E.S.

Neurological examination and patients group classification; L.J.A., A.S.W., W.M. , F.D., C.S., B. intellectual contribution to study design and review of manuscript

\section{References}

1. Lacey M. Nodding disease: mystery of southern Sudan. Lancet Neurol 2003;2:714.

2. Tumwine J, Vandemaele K, Chungong S, Richer M, Anker M, Ayana Y, et al. Clinical and epidemiologic characteristics of nodding syndrome in Mundri County, southern Sudan. Afr Health Sci 2012;12:242-8.

3. Nyungura JL, Akim T, Lako A, Gordon A, Lejeng L, William G. Investigation into the Nodding syndrome in Witto Payam, Western Equatoria State, 2010. South Sudan Med J 2011;4:3-6.

4. Aall-Jilek LM. Epilepsy in the Wapogoro tribe in Tanganyika. Acta Psychiatr Scand 1965;41:57-86.

5. Winkler AS, Friedrich K, König R, Meindl M, Helbok $\mathrm{R}$, Unterberger I, et al. The head nodding syndrome-clinical classification and possible causes. Epilepsia 2008;49:2008-15.

6. Winkler AS, Wallner B, Friedrich K, Pfausler B, Unterberger I, Matuja W, et al. A longitudinal study on nodding syndrome-a new African epilepsy disorder. Epilepsia 2014;55:86-93.

7. Spencer PS, Palmer VS, Jilek-Aall L. Nodding syndrome: origins and natural history of a longstanding epileptic disorder in sub-Saharan Africa. Afr Health Sci 2013;13:176- 82 .

8. Kaiser C, Benninger C, Asaba G, Mugisa C, Kabagambe G, Kipp W, et al. Clinical and electro-clinical classification of epileptic seizure in west Uganda. Bull Soc Pathol Exot 2000;93:255-9.

9. Sejvar JJ, Kakooza AM, Foltz JL, Makumbi I, AtaiOmoruto AD, Malimbo M, et al. Clinical, neurological, 
and electrophysiological features of nodding syndrome in Kitgum, Uganda: an observational case series. Lancet Neurol 2013;12:166-74.

10. Cross JH. Nodding syndrome--a challenge for African public health. Lancet Neurol 2013;12:125-6.

11. Idro R, Opoka RO, Aanyu HT, Kakooza-Mwesige A, Piloya-Were T, Namusoke $H$, et al. Nodding syndrome in Ugandan children--clinical features, brain imaging and complications: a case series. BMJ Open 2013;3.

12. Winkler AS, Friedrich K, Meindl M, Kidunda A, Nassri A, Jilek-Aall L, et al. Clinical characteristics of people with head nodding in southern Tanzania. Trop Doct 2010;40:173-5.

13. WHO. Nodding Syndrome meeting, researchers agree on case definition and establish research agenda 2012.

14. Dowell SF, Sejvar JJ, Riek L, Vandemaele KAH, Lamunu M, Kuesel AC, et al. Nodding syndrome. Emerg Infect Dis 2013;19:1374-84.

15. WHO, Uganda G of. Uganda adopts a multi-sectoral response to nodding syndrome. Press Release 1/2012 2012:1-2.

16. Spencer PS, Vandemaele K, Richer M, Palmer VS, Chungong S, Anker M, et al. Nodding syndrome in Mun- dri county, South Sudan: environmental, nutritional and infectious factors. Afr Health Sci 2013;13:183-204.

17. Winkler AS, Friedrich K, Velicheti S, Dharsee J, König R, Nassri A, et al. MRI findings in people with epilepsy and nodding syndrome in an area endemic for onchocerciasis: an observational study. Afr Health Sci 2013;13:529-40.

18. Foltz JL, Makumbi I, Sejvar JJ, Malimbo M, Ndyomugyenyi R, Atai-Omoruto AD, et al. An Epidemiologic Investigation of Potential Risk Factors for Nodding Syndrome in Kitgum District, Uganda. PLoS One 2013;8:e66419.

19. Vincent A, Bien CG. Anti-NMDA-receptor encephalitis: a cause of psychiatric, seizure, and movement disorders in young adults. Lancet Neurol 2008;7:1074-5.

20. Bergey GK. Autoantibodies in the patient with drug-resistant epilepsy: are we missing a treatable etiology? Arch Neurol 2012;69:565-6.

21. König R, Nassri A, Meindl M, Matuja W, Kidunda AR, Siegmund V, et al. The role of Onchocerca volvulus in the development of epilepsy in a rural area of Tanzania. Parasitology 2010;137:1559-68.

22. Kuchinskas EJ, Horvath A, DU Vigneaud V. An anti-vitamin B6 action of L-penicillamine. Arch Biochem Biophys 1957;68:69-75. 